\title{
Influencia de la automatización de ensayos de flujo luminoso total en el cálculo de incertidumbre combinada y en el tiempo de ejecución del ensayo
}

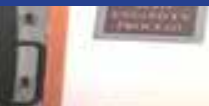

Influence of the automation of the total luminous flux test on the calculation of the combined uncertainty and the period of execution of the test

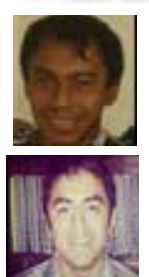

\section{De la Bastida Ronny}

Escuela Politécnica Nacional (EPN) e-mail: ronny.delabastida@epn.edu.ec

\section{Velásquez Carlos}

Instituto Nacional de Eficiencia Energética y Energías (INER) e-mail: carlos.velasquez@iner.gob.ec

\section{Resumen}

El cálculo de la incertidumbre combinada de ensayos de flujo luminoso realizados en una esfera integradora es importante para la acreditación bajo los estándares de la norma ISO/IEC 17025. Los valores de los equipos de medición usados dentro del ensayo debían ser registrados de manera manual por el operador, por lo cual se implementó un sistema de adquisición de los datos automático mediante la integración de los equipos en una aplicación desarrollada en LabVIEW que incluye el cálculo de la incertidumbre basado en el modelo desarrollado por el mismo laboratorio y la generación de informes. El presente trabajo analiza la influencia de la automatización del proceso de adquisición, análisis y uso de los datos para el cálculo de la incertidumbre, comparando un ensayo designado de una lámpara de alta descarga usando el sistema manual y el sistema automatizado además de la reducción del tiempo del ensayo para cubrir una mayor demanda

Palabras clave: automatización; esfera integradora; flujo luminoso; incertidumbre combinada

Abstract

The calculation of the combined uncertainty of luminous flux tests carried out in an integrating sphere is a very important value for the accreditation of INER's Lighting Laboratory under the ISO/IEC 17025 standard. The values of the measurement devices used within the test should be registered manually by the operator, for which an automatic data acquisition system was implemented by integrating the devices into an application developed in LabVIEW that includes a real-time analysis of the luminous flux stabilization of the lamp and the calculation of the uncertainty based on the model developed by INER's Lighting Laboratory. The present work analyzes the influence of the automation of the process of acquisition, analysis and use of the data for the calculation of the uncertainty, comparing a designated test of a high discharge lamp using the manual system and the automated system for the calculation of the combined uncertainty of the luminous flux test within an integrating sphere.

Keywords: automation; integrating sphere; luminous flux; combined uncertainty.

\section{Introducción}

En el Ecuador el alumbrado público representa un $7.70 \%$ del rubro de la energía facturada del país (Agencia de Regulación y Control de Electricidad, 2016) según la última estadística de la Agencia de Regulación y Control de Electricidad (ARCONEL). Para el estudio de mejoramiento de este campo el
Instituto Nacional de Eficiencia Energética y Energías Renovables (INER) creó un Laboratorio de Luminotecnia que es un Organismo Evaluador de Conformidad (OEC), encargado de realizar ensayos de flujo luminoso a lámparas de descarga de alta intensidad (HID) (Ministerio de Industrias y Productividad, 2016). 
La eficiencia energética de lámparas es una de las magnitudes usadas para establecer una comparativa entre dos fuentes de luz de las mismas características, para lo cual es necesario obtener el flujo luminoso total de la lámpara y las magnitudes eléctricas de consumo de la misma (Espín, et al., 2016). Para obtener resultados confiables se realiza los ensayos dentro de un ambiente de temperatura controlada, usando una Esfera Integradora con el método recomendado por la Illuminating Engineer Society of North America (IESNA) (Illuminating Engineering Society, 2017). Este método exige la medición de una lámpara de referencia (patrón de flujo luminoso total), una lámpara de prueba, dado que es una medición relativa y la medición de absorbancia de ambas lámparas empleando una lámpara auxiliar del tipo halógena incandescente.

Los ensayos de flujo luminoso son realizados bajo un sistema de gestión conforme a la normativa ISO/ IEC 17025, que es la referencia internacional para garantizar la competencia técnica en ensayos de cualquier magnitud (Instituto Ecuatoriano de Normalización , 2015). Un parámetro indispensable de la norma es el cálculo de incertidumbre del ensayo, que en este estudio se realiza mediante un modelo matemático implementado por el mismo laboratorio y descrito en las Ec. 1, Ec. 2 y Ec. 3.

$$
\begin{array}{cc}
\emptyset=\emptyset_{R} \frac{v}{v_{R}} \frac{\Phi_{A R}}{\Phi_{A}}\left(\frac{v \cdot c_{v}}{V_{0}}\right)^{m_{v}}\left(\frac{U_{R} * c_{R}}{R \cdot J_{R}}\right)^{m_{j R}}\left(\frac{\text { cors } S_{R}}{\operatorname{corS}}\right) & \text { Ec. (1) } \\
\operatorname{cor} S=1+\alpha \Delta \mathrm{T}-\Delta \mathrm{sf}-\gamma \Delta \mathrm{t}-\mathrm{S} & \text { Ec. (2) } \\
\operatorname{corS}_{R}=1+\alpha_{R} \Delta \mathrm{T}_{\mathrm{R}}-\Delta \mathrm{Sf}_{\mathrm{R}}-\gamma_{R} \Delta \mathrm{t}_{\mathrm{R}}-\mathrm{S}_{\mathrm{R}} & \text { Ec. (3) }
\end{array}
$$

El significado de las variables puede ser observado en la Tabla 1 y la correspondiente deducción de las mismas se encuentra en el trabajo (Velásquez \& Espín , 2015).

La automatización de la toma de datos durante el ensayo influye directamente en su tiempo de ejecución y en el cálculo de incertidumbre del resultado. Realizar este proceso es importante para la mejora de las capacidades operativas del laboratorio y una muestra de la mejora continua, exigida por los estándares de calidad.

El objetivo del presente artículo es cuantificar la influencia de un determinado proceso de ingeniería de automatización en la incertidumbre y tiempos de ensayo, esto ayudará a laboratorios paralelos a tener perspectiva sobre la mejora de sus procedimientos. Para este fin se ha ensayado una lámpara HID de sodio con el método usual del laboratorio y el método automatizado, analizando sus respetivos resultados.

\begin{tabular}{|c|c|}
\hline Símbolo & Variable \\
\hline$\emptyset$ & Flujo luminoso teórico \\
\hline$\emptyset_{R}$ & Flujo luminoso lámpara de referencia \\
\hline$v$ & $\begin{array}{l}\text { Señal del fotosensor de lámpara de } \\
\text { prueba }\end{array}$ \\
\hline$v_{R}$ & $\begin{array}{l}\text { Señal del fotosensor de lámpara de } \\
\text { referencia }\end{array}$ \\
\hline$\emptyset_{A R}$ & $\begin{array}{l}\text { Flujo luminoso de absorbancia lámpara } \\
\text { de referencia }\end{array}$ \\
\hline$\emptyset_{A}$ & $\begin{array}{l}\text { Flujo luminoso de absorbancia lámpara } \\
\text { de prueba }\end{array}$ \\
\hline $\mathrm{V}$ & Voltaje de lámpara de prueba \\
\hline$c_{V}$ & Factor de calibración voltaje AC \\
\hline$V_{0}$ & Voltaje nominal lámpara de prueba \\
\hline$m_{v}$ & $\begin{array}{l}\text { Exponente de variación corriente-flujo de } \\
\text { lámpara de prueba }\end{array}$ \\
\hline$U_{R}$ & $\begin{array}{l}\text { Voltaje de derivación de lámpara de } \\
\text { referencia }\end{array}$ \\
\hline$c_{R}$ & Factor de calibración del multímetro \\
\hline$R$ & Resistencia de derivación \\
\hline$J_{R}$ & Corriente nominal lámpara de referencia \\
\hline $\operatorname{corS}_{R}$ & $\begin{array}{l}\text { Factor de corrección lámpara de } \\
\text { referencia }\end{array}$ \\
\hline cors & Factor de corrección lámpara de prueba \\
\hline$\alpha$ & $\begin{array}{l}\text { Coeficiente de temperatura ambiental de } \\
\text { lámpara de prueba }\end{array}$ \\
\hline$\Delta \mathrm{T}$ & $\begin{array}{l}\text { Diferencia de temperatura ambiental } \\
\text { lámpara de prueba }\end{array}$ \\
\hline$\Delta \mathrm{sf}$ & Factor espacial lámpara de prueba \\
\hline$\gamma$ & $\begin{array}{l}\text { Coeficiente de envejecimiento lámpara de } \\
\text { prueba }\end{array}$ \\
\hline$\Delta \mathrm{t}$ & $\begin{array}{l}\text { Tiempo de estabilización lámpara de } \\
\text { prueba }\end{array}$ \\
\hline $\mathrm{S}$ & Luz no deseada lámpara de prueba \\
\hline$\Delta \mathrm{sf}$ & Factor espacial lámpara de prueba \\
\hline$\gamma$ & $\begin{array}{l}\text { Coeficiente de envejecimiento lámpara de } \\
\text { prueba }\end{array}$ \\
\hline$\Delta \mathrm{t}$ & $\begin{array}{l}\text { Tiempo de estabilización lámpara de } \\
\text { prueba }\end{array}$ \\
\hline$\alpha_{R}$ & $\begin{array}{l}\text { Coeficiente de temperatura ambiental } \\
\text { lámpara de referencia }\end{array}$ \\
\hline$\Delta T_{R}$ & $\begin{array}{l}\text { Diferencia de temperatura ambiental } \\
\text { lámpara de referencia }\end{array}$ \\
\hline$\Delta s f_{R}$ & Factor espacial lámpara de referencia \\
\hline$\gamma_{R}$ & $\begin{array}{l}\text { Coeficiente de envejecimiento lámpara de } \\
\text { referencia }\end{array}$ \\
\hline$\Delta \mathrm{t}_{\mathrm{R}}$ & $\begin{array}{l}\text { Tiempo de estabilización lámpara de } \\
\text { referencia }\end{array}$ \\
\hline $\mathrm{S}_{\mathrm{R}}$ & Luz no deseada lámpara de referencia \\
\hline
\end{tabular}

Tabla 1. Símbolos y nombres de las variables del modelo de in-certidumbre de la esfera integradora del Laboratorio de Lumino-tecnia del INER

\section{Metodología}

El ensayo de flujo luminoso consistía en la toma manual de datos por parte del personal del laboratorio para el registro de mediciones en archivos auxiliares, que permitían calcular la incertidumbre 
combinada del ensayo. Los equipos usados en la Fig. 1 corresponden a un fotómetro PR-200D con cabeza fotométrica corregida con la función de visión fotópica $(\mathrm{V}(\lambda))$, un multímetro Yokogawa WT310 que obtiene los parámetros eléctricos consumidos por la fuente de luz, una fuente de corriente continua ITECH IT6154 que energiza la lámpara para la medición de absorbancia y un termohigrómetro MSR255 que mide la temperatura ambiental del cuarto. Todos los equipos se encuentran calibrados en laboratorios acreditados con la norma ISO/IEC 17025 (Instituto Ecuatoriano de Normalización , 2015).

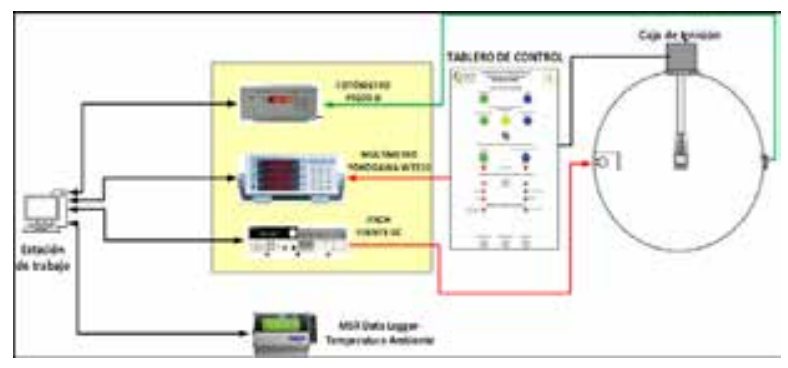

Figura 1. Sistema de medición en una esfera integradora. Fuente (De La Bastida, et al., 2017)

Se diseñó e implantó un sistema de adquisición automática que registró los datos de los equipos y realizó el cálculo de incertidumbre sin intervención del operador mediante una aplicación desarrollada en LabVIEW de acuerdo a lo expuesto en (De La Bastida, et al., 2017). La aplicación ejecuta flujos de programación paralelos para realizar tareas de análisis y muestreo de manera continua de las variables presentes en el ensayo (De La Bastida, et al., 2017).

El modelo de incertidumbre emplea los datos fotométricos y eléctricos registrados en las diversas etapas del ensayo. Los registros usados para el cálculo de la incertidumbre son aquellos verificados de acuerdo a los criterios de estabilización de la lámpara según la IES LM 51-13 (Illuminating Engineering Society of North America, 2013). El esquema de adquisición de datos y cálculo de las variables se puede observar en la Fig. 2.

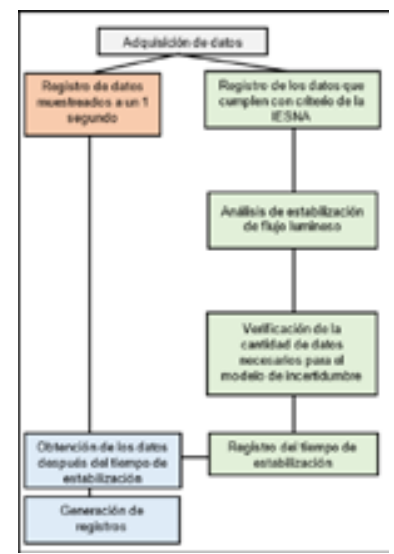

Figura 2. Esquema del proceso de adquisición de datos para el análisis de estabilidad de flujo y registro de los datos de forma automática
Una vez automatizada la toma de datos, se procedió a realizar el ensayo según el método manual y automatizado, ambos de acuerdo a los procesos descritos en la Fig. 3.

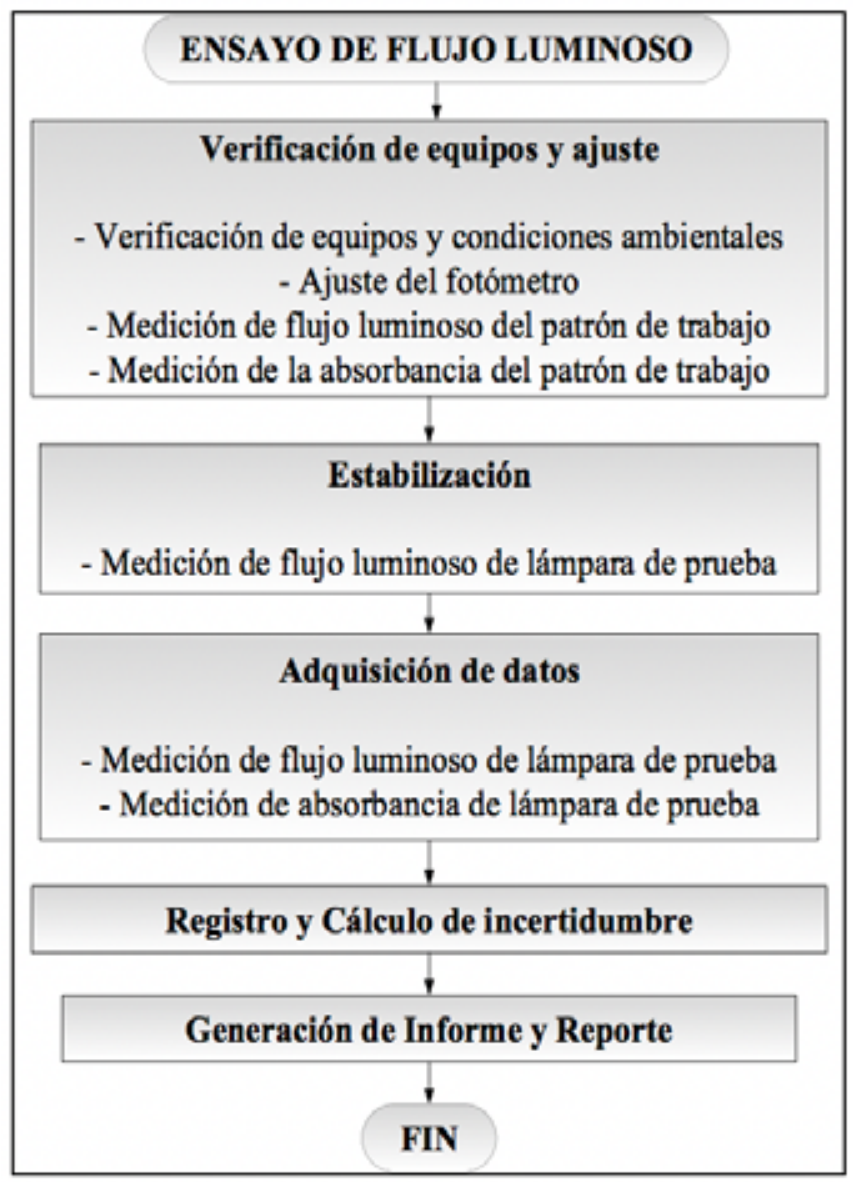

Figura 3. Esquema del proceso de ensayo de flujo luminoso para una lámpara usando una esfera integradora

Se calculó la incertidumbre en ambos ensayos y se procedió a comparar los resultados de cada variable, adicionalmente se tomaron medidas de tiempo para cada fase en cada método y se calculó la reducción total de tiempo.

\section{Resultados y discusión}

Las magnitudes medidas que intervienen en el cálculo de incertidumbre son la temperatura ambiental, flujo luminoso y voltaje consumido por la fuente de luz. Cada una de estas se monitorearon durante cada uno de los procesos realizados durante el ensayo de la lámpara HID.

Para el inicio de ambas pruebas se verificó que las condiciones ambientales se mantengan dentro de lo que dicta el método (Illuminating Engineering Society of North America, 2013). 


\section{Influencia en el cálculo de la incertidumbre combinada}

Temperatura ambiental

El comportamiento de la temperatura durante el ensayo de la lámpara de prueba y lámpara de referencia se observan en la Fig. 4 y Fig. 5 respectivamente.

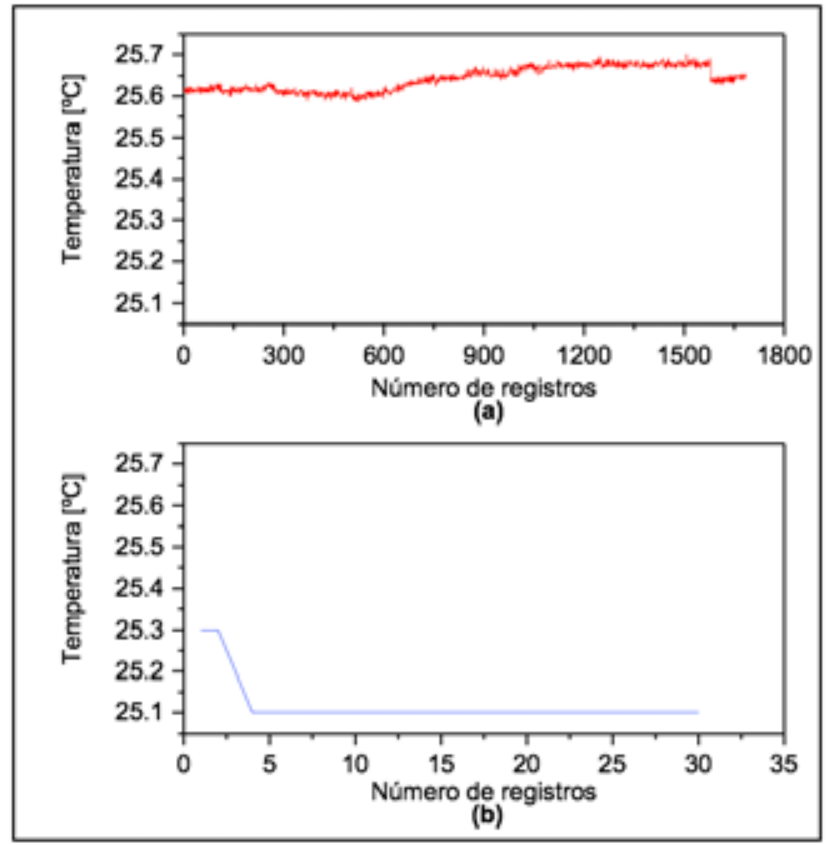

Figura 4. Evolución de toma de datos de temperatura durante el ensayo de lámpara de prueba (a) sistema automatizado y (b) sistema manual

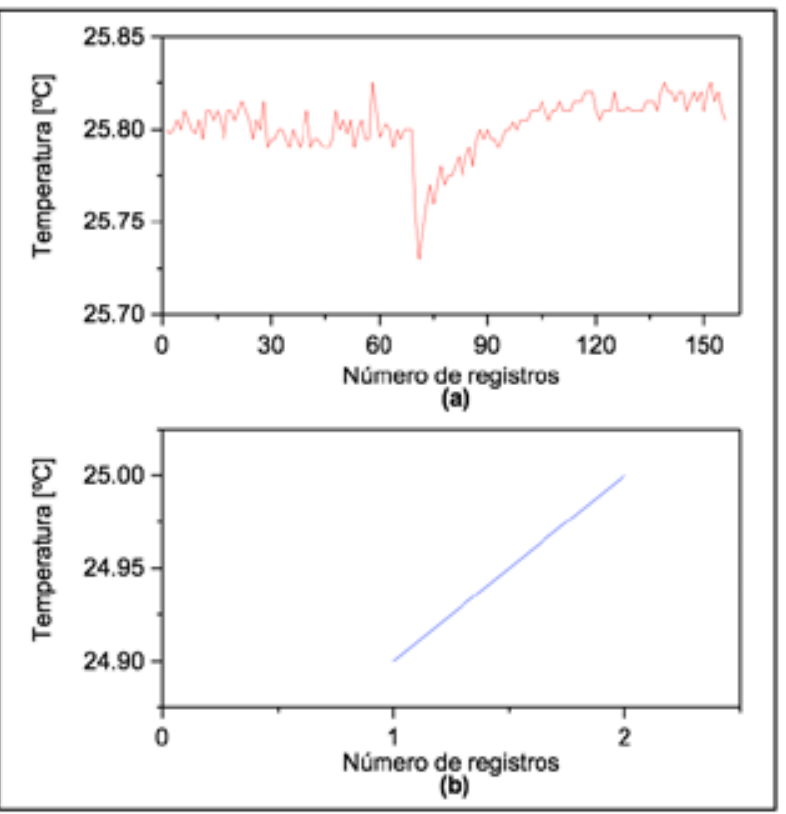

Figura 5. Evolución de toma de datos de temperatura durante la medición de flujo luminoso de lámpara de referencia (a) sistema automatizado y (b) sistema manual

La Figura 4 (a) y Figura 5 (a) correspondientes a la adquisición con sistema automático muestran una mayor cantidad de información de la evolución de temperatura durante el ensayo de cada lámpara. A diferencia de la Fig. 4 (b) y Fig. 5 (b) correspondien- tes a la adquisición de manera manual que muestran poco detalle en la evolución de temperatura. Esto se debe a que la capacidad del sistema automatizado permite la adquisición de 1 registro cada segundo, en contraste con el sistema manual, la adquisición de datos del Termohigrómetro que está configurado a una tasa de muestreo de 1 registro cada minuto.

En la Tabla 2 se observa que el número de registros obtenidos con el sistema automatizado en ambos ensayos tiene un incremento de más del doble de datos obtenidos por el sistema manual.

Tabla 2. Número de registros de temperatura ambiental obteni-dos usando el sistema manual y el sistema automático

\begin{tabular}{|c|c|c|}
\hline Lámpara & Manual & Automático \\
\hline Prueba & 30 & 1685 \\
\hline Referencia & 2 & 156 \\
\hline
\end{tabular}

La diferencia en la cantidad de registros entre las lámparas de prueba y referencia se da por el tiempo de medición. La primera es aproximadamente 30 minutos y en la segunda 2 minutos.

\section{Medición de flujo luminoso}

La medición de flujo luminoso es el objetivo del ensayo, su evolución en las lámparas de prueba y referencia, medidas con el fotómetro PR-200D se observan en la Fig. 6 y Fig. 7 respectivamente.

Particularmente, en la Figura 6 (a) aparecen 8 picos que se apartan de la tendencia de los otros datos del flujo luminoso, este fenómeno no es más que un error de lectura propio del fotómetro PR-200D que independientemente con qué método se lo haga aparece de manera aleatoria. Estas medidas no son consideradas por el operador cuando se usa el sistema manual, por considerarlas un error de medida del dispositivo. En el sistema automatizado, las lecturas erróneas del dispositivo son minimizadas debido a que en el promedio de la gran cantidad de datos su ponderación tiende a cero.

En la Tabla 3 se muestra que la cantidad de registros obtenidos usando el sistema automatizado son 75 veces mayores a los datos obtenidos por el operador usando el sistema manual para la lámpara de prueba, y casi 3 veces más para la lámpara de referencia. Esto nuevamente comprueba la ventaja de la capacidad de registro del sistema automatizado sobre el sistema manual. 

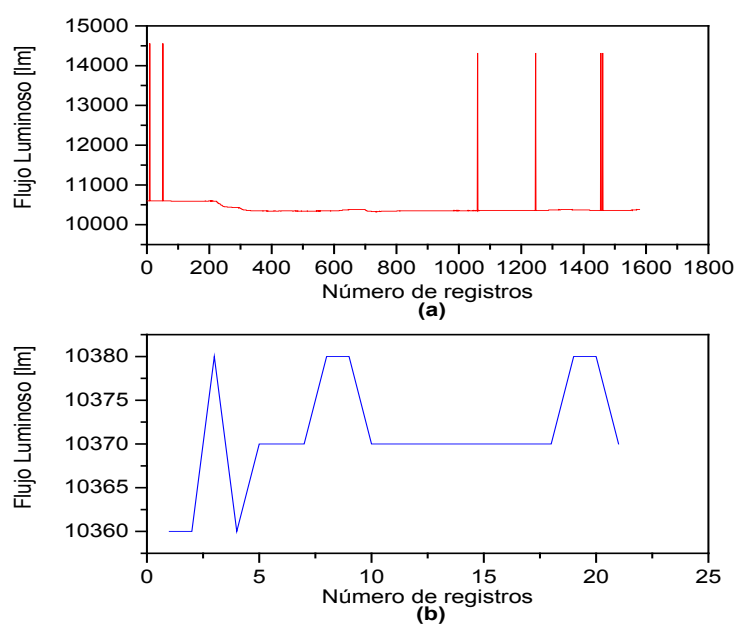

Figura 6. Evolución del flujo luminoso de la lámpara de prueba de descarga de alta intensidad (a) sistema automatizado y (b) sistema manual
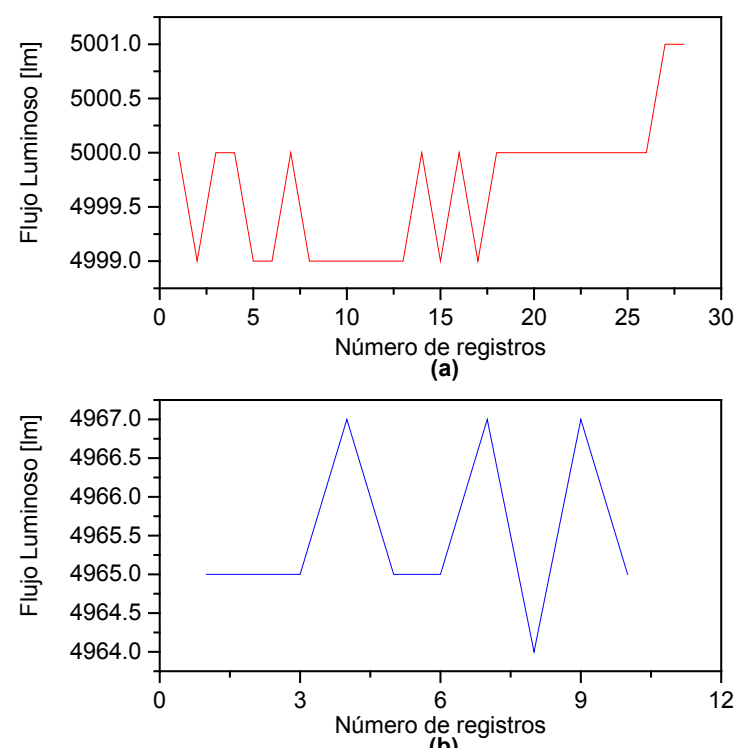

(b)

Figura 7. Evolución del flujo luminoso de la lámpara de referencia (a) sistema automatizado y (b) sistema manual

Tabla 3. Número de registros de temperatura ambiental obtenidos usando el sistema manual y el sistema automático

\begin{tabular}{|c|c|c|}
\hline Lámpara & Manual & Automático \\
\hline Prueba & 21 & 1579 \\
\hline Referencia & 10 & 28 \\
\hline
\end{tabular}

\section{Medición de absorbancia}

La Figura 8 y Figura 9 corresponden a la medición de absorbancia de flujo luminoso de las lámparas de prueba y referencia respectivamente. Se puede observar una evolución del flujo luminoso mucho más definida por la cantidad de datos adquiridos con el sistema automatizado.
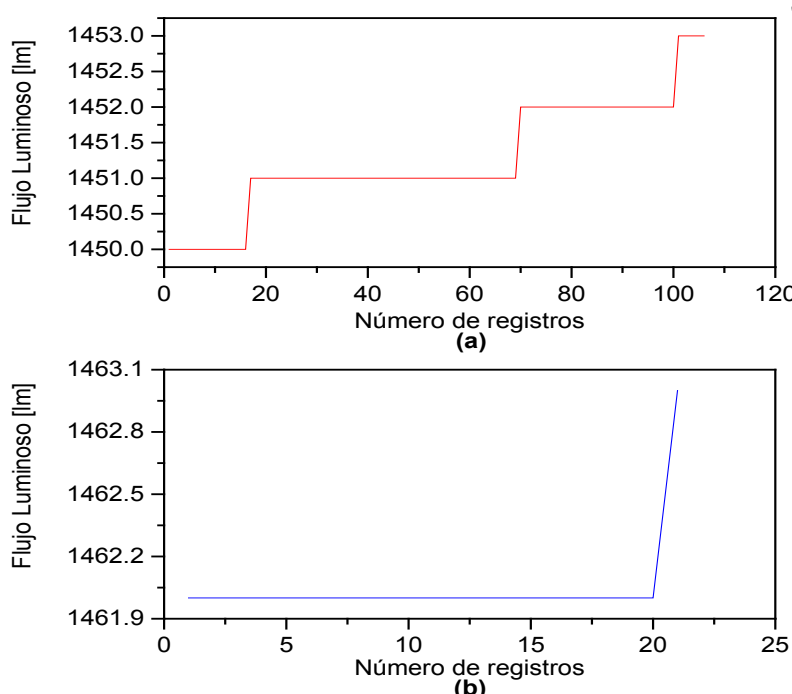

(b)

Figura 8. Evolución del flujo luminoso de la medición de absorbancia de la lámpara de prueba de descarga de alta intensidad (a) sistema automatizado y (b) sistema manual

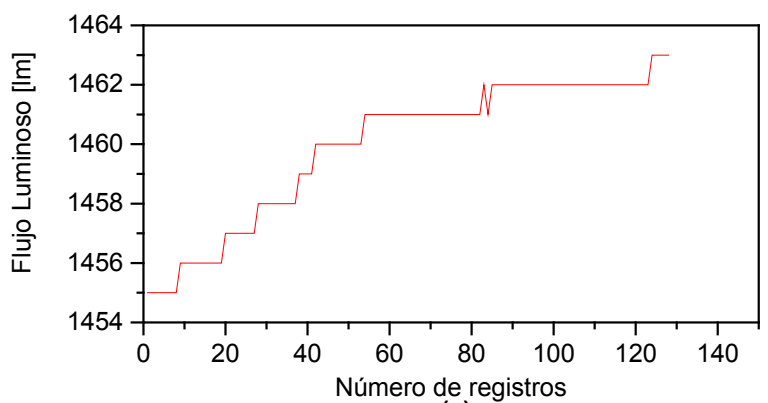

(a)

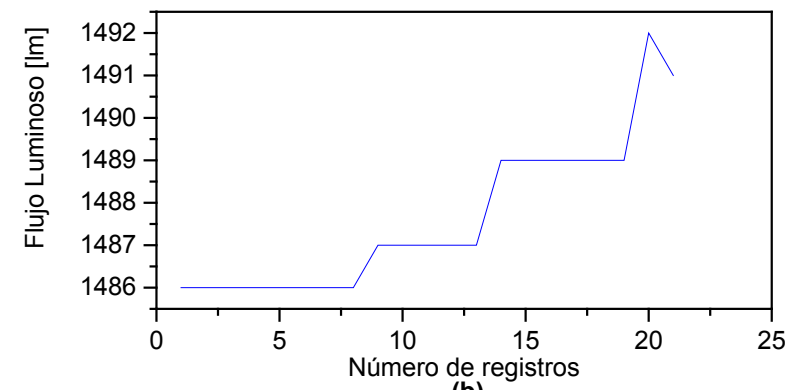

(b)

Figura 9. Evolución del flujo luminoso de la medición de absorbancia de la lámpara de referencia (a) sistema automatizado y (b) sistema manual

La Tabla 4 muestra que el número de registros obtenidos de la medición de absorbancia de las lámparas de prueba y referencia usando el sistema automatizado es 5 veces mayor a la cantidad de datos obtenidos de manera manual.

En la medición de absorbancia de ambas lámparas usando el método manual o automático, el número de registros es similar, debido a que la medición de absorbancia, independientemente de la lámpara, tiene un tiempo de medición similar. 
Tabla 4. Número de registros de la absorbancia obtenidos usando el sistema manual y el sistema automático

\begin{tabular}{|c|c|c|}
\hline Lámpara & Manual & Automático \\
\hline Prueba & 21 & 106 \\
\hline Referencia & 21 & 128 \\
\hline
\end{tabular}

\section{Medición de voltaje}

En la Figura 10 se muestra el comportamiento del voltaje durante la medición de la lámpara de prueba, observándose variaciones dentro de la ejecución del ensayo. El detalle de variación de voltaje es mucho más definido con el sistema automático, debido a que la cantidad de información registrada es 75 veces mayor que usando el sistema manual.

La Tabla 5 evidencia nuevamente la ventaja en la capacidad de monitoreo del sistema automático con lo cual el operador tiene mayor información del comportamiento eléctrico de la medición de la lámpara.

Tabla 5. Número de registros del registro de voltaje consumido por la lámpara de prueba usando el sistema manual y el sistema automático

\begin{tabular}{|c|c|c|}
\hline Lámpara & Manual & Automático \\
\hline Prueba & 21 & 1579 \\
\hline
\end{tabular}

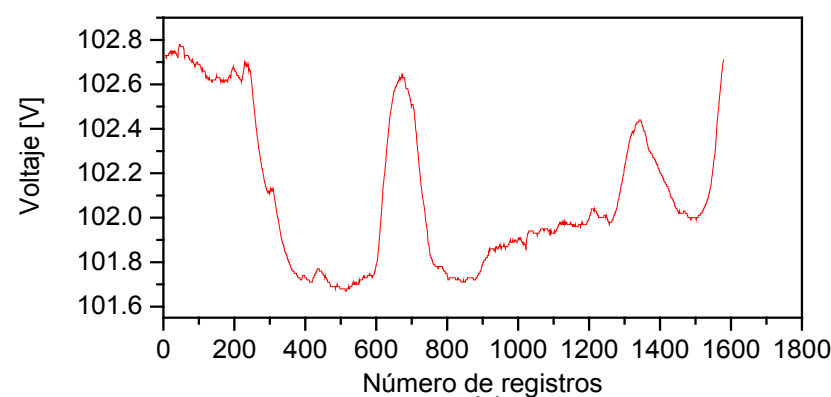

(a)

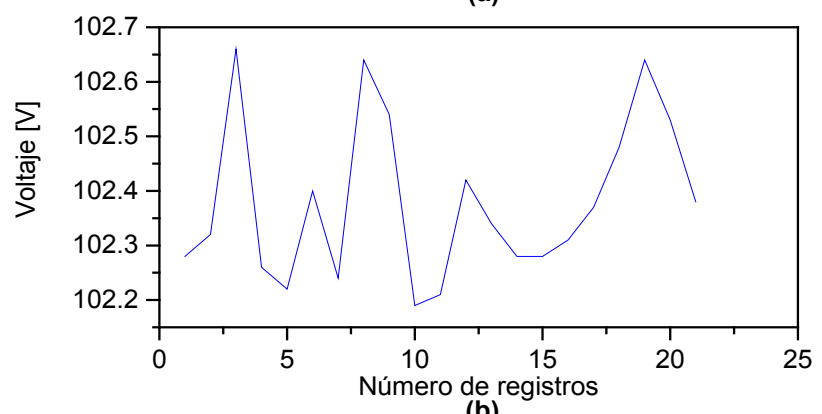

(b)

Figura 10. Evolución del voltaje consumido por la lámpara de prueba de descarga de alta intensidad (a) sistema automatizado y (b) sistema manual

\section{Parámetros del modelo de incertidumbre}

La influencia de la automatización se puede observar en el cambio de los valores correspondientes a los parámetros calculados por el modelo de incertidumbre y mostrados en la Tabla 6. Donde y son las temperaturas promedio de las lámparas de referencia y prueba respectivamente. GDL son los grados de libertad efectivos y $\mathrm{k}$ es el factor de cobertura del ensayo. Los demás parámetros corresponden a la Ec. (1) y se las puede encontrar en la Tabla 1.

En la diferencia de temperatura ambiental, tanto en la lámpara de referencia y la lámpara de prueba, se observa que la consecuencia de usar el método automatizado implica tener una variación 3 y 7 veces menor respectivamente.

Los valores de flujo luminoso de la lámpara de prueba obtenidos con los dos sistemas difieren en $0.36 \%$, esto a pesar que el método automatizado incluye los errores de medida del dispositivo presentados en la Fig. 6 (a). Estos aportan un error de 38.89 lúmenes $(\mathrm{Im})$, el cual es un valor despreciable por el rango de flujo medido de la lámpara HID, que en este caso es aproximadamente $10000 \mathrm{~lm}$. Hay que mencionar que, si uno de estos picos coincidiera con la toma de un dato por el método manual causaría un incremento en el promedio de la medición.

Otra de las consecuencias de la automatización está en el número de grados de libertad efectivo, que es cinco veces mayor, lo que asegura un factor de cobertura igual a 2 .

Finalmente, el valor de la incertidumbre combinada en el sistema manual difiere en la tercera cifra decimal del porcentaje con respecto al método automático, es decir $0.01 \%$. Sin embargo, hablar de 3 cifras decimales porcentuales en una medición de flujo luminoso total carece de sentido físico, por tanto, tomando únicamente un decimal de la incertidumbre, conforme a la norma ISO/IEC 17025, su valor es de $7.5 \%$ en ambos casos. Esto implica que la toma de datos de forma automática verifica que la incertidumbre no se encuentra sobreestimada o subestimada, una forma distinta de tomar datos genera la misma incertidumbre de ensayo. 
Tabla 6. Valores de los parámetros calculados dentro del modelo de incertidumbre usando el sistema manual y el sistema auto-mático.

\begin{tabular}{|c|c|c|c|}
\hline & Parámetro & Sistema Manual & Sistema Automático \\
\hline \multirow{4}{*}{$\begin{array}{c}\text { Lámpara de } \\
\text { referencia }\end{array}$} & & 24.93 & 25.82 \\
\hline & & 0.0345 & 0.01104 \\
\hline & & 4965 & 4999.67 \\
\hline & & 1487.61 & 1459.94 \\
\hline \multirow{5}{*}{$\begin{array}{l}\text { Lámpara de } \\
\text { prueba }\end{array}$} & & 25.12 & 25.65 \\
\hline & & 0.1897 & 0.02539 \\
\hline & & 10371.95 & 10409.84 \\
\hline & & 1462.05 & 1451.25 \\
\hline & v & 102.38 & 102.10 \\
\hline \multirow{4}{*}{$\begin{array}{c}\text { Parámetros } \\
\text { calculados del } \\
\text { modelo de } \\
\text { incertidumbre }\end{array}$} & $\begin{array}{l}\text { GDL efec- } \\
\text { tivos }\end{array}$ & 50 & 288 \\
\hline & K & 2 & 2 \\
\hline & & 10629.55 & 10466.15 \\
\hline & $\begin{array}{c}\text { Incerti- } \\
\text { dumbre } \\
\text { combinada } \\
{[\%]}\end{array}$ & 7.478742 & 7.4801145 \\
\hline
\end{tabular}

\section{Tiempos de operación}

La optimización del tiempo utilizado por ensayo es importante para mejorar la productividad. La Figura 11 muestra los indicadores de tiempos estimados en cada una de los procesos del ensayo de flujo luminoso usando el sistema manual y el sistema automático, estas etapas son las mismas que se encuentran en la Fig. 3.

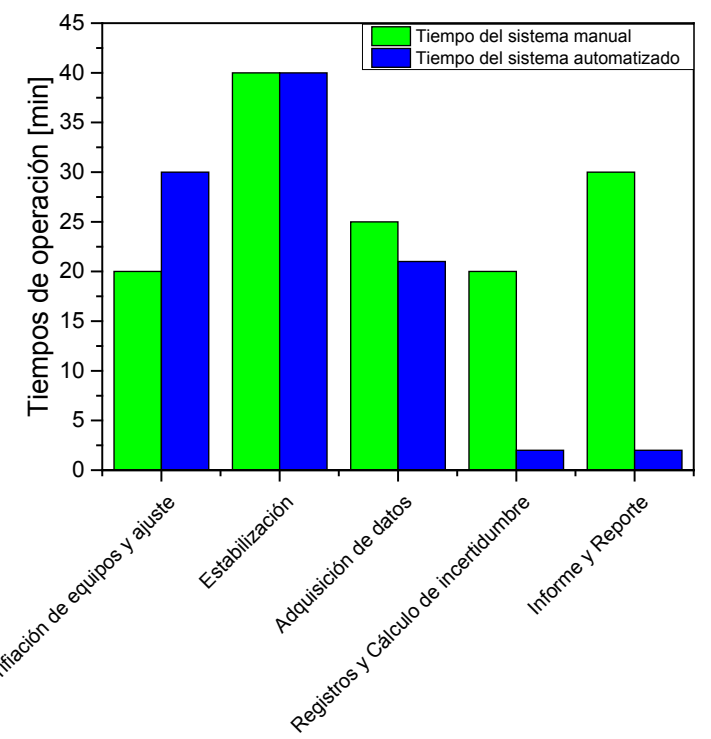

Figura 11. Tiempos de operación de cada una de los procesos de ensayo de flujo luminoso de una lámpara HID usando el sistema manual y el sistema automatizado

Se observa que durante la primera etapa de verificación y ajuste usando el sistema automatizado se requiere de un mayor tiempo, esto se debe al pro- cedimiento de ajuste del fotómetro del laboratorio adelantando a las características del sistema automatizado.

Durante el procedimiento de estabilización de la lámpara, ambos sistemas requieren el mismo debido a que el ítem de ensayo es del mismo tipo (tecnología de lámpara), sin embargo, es importante destacar que pueden existir lámparas con bondades técnicas mejores que permitan alcanzar la estabilidad en menor tiempo que el aproximado, en este caso el método manual tardará el mismo tiempo aproximado y el automatizado un tiempo menor ya que es capaz de detectar esta estabilización en tiempo real.

La tercera etapa muestra una reducción relativamente pequeña, este período se trata de la toma de datos (uno por minuto). El método manual depende del operador que demora 25 minutos en tomar 21 datos, el automatizado se demora solo 21 minutos tomando 1579 datos.

Las dos últimas etapas muestran una reducción total de tiempos, en el cálculo de incertidumbre y elaboración de informe que en el método manual el operador está obligado a realizar siempre en un mínimo de 20 y 30 minutos, el método automático lo realiza en menos de un minuto.

La Tabla 7 muestra los porcentajes de reducción en cada una de las etapas dentro del ensayo de flujo luminoso de una lámpara HID, donde en la primera etapa se tiene un incremento en el tiempo en un 50 $\%$ y desde la etapa de adquisición se ve un aumento del porcentaje de reducción del tiempo usando el sistema automatizado.

Tabla 7. Porcentaje de reducción de tiempos usando el sistema automatizado para ensayos de flujo luminoso

\begin{tabular}{|c|c|}
\hline Fase del ensayo & $\begin{array}{c}\text { Porcentaje de reducción } \\
\text { de tiempo }\end{array}$ \\
\hline $\begin{array}{c}\text { Verificación de equipos y } \\
\text { ajuste }\end{array}$ & $-50 \%$ \\
\hline Estabilización & $0 \%$ \\
\hline Adquisición de datos & $16 \%$ \\
\hline $\begin{array}{c}\text { Registros y Cálculo de in- } \\
\text { certidumbre }\end{array}$ & $90 \%$ \\
\hline Informe y Reporte & $93 \%$ \\
\hline
\end{tabular}

La reducción del tiempo total de un ensayo se puede observar en la Fig. 12, donde se evidencia una reducción del 30\%, o lo que equivale una disminu- 
ción de 40 minutos por cada uno de los ensayos usando el sistema automatizado.

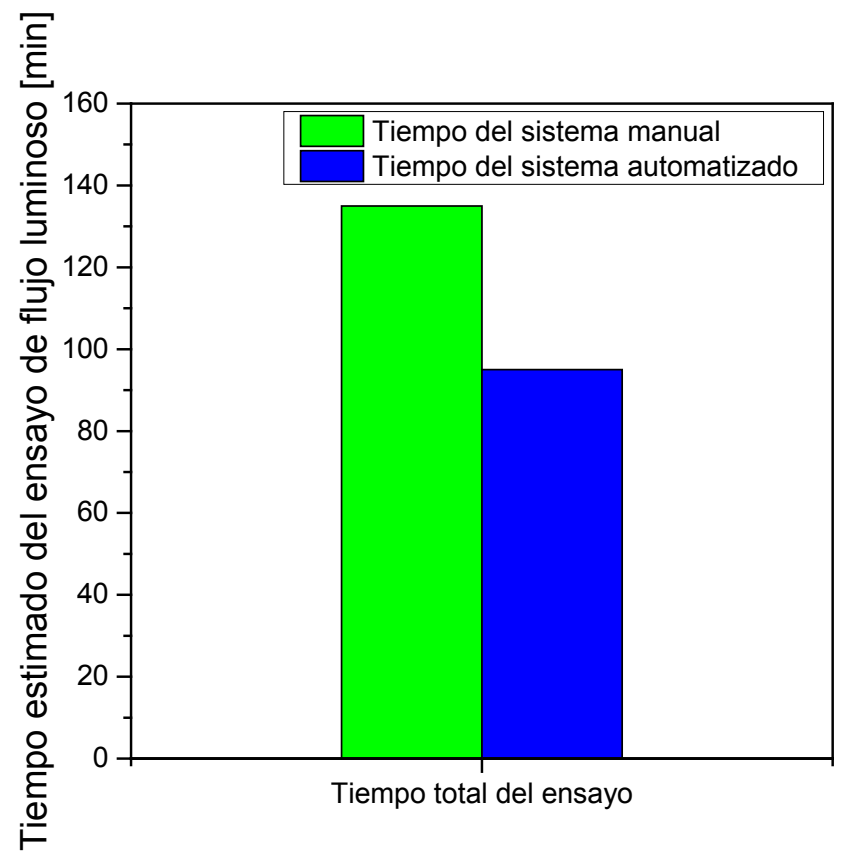

Figura 12. Tiempos totales estimados de duración de un ensayo de una lámpara HID usando el sistema manual y el sistema automático

\section{Conclusiones}

La automatización de la adquisición de los datos y cálculo de la incertidumbre combinada durante los ensayos de flujo luminoso mejoran el proceso, existe menos carga de análisis para el operador y evita errores de medición inherentes al ser humano.

Los errores propios de los dispositivos siguen presentes durante el proceso y su influencia en el valor promedio ha sido reducida por la gran cantidad de datos registrados, en particular los valores de flujo luminoso. La diferencia de la temperatura ambiental también se ha reducido por la cantidad de datos y el factor de cobertura igual a 2 ha sido asegurado.

El valor de la incertidumbre no se ha visto afectado, pero se debe tomar en cuenta que usando el método automatizado incluimos todos los errores de medición presentes (como los picos de flujo) y obtenemos los mismos resultados, y mediante el registro manual está la posibilidad que al reportar un error de medición los valores finales se vean comprometidos.

La reducción del tiempo de ensayo usando el sistema automático permite concluir que se puede incrementar la productividad del laboratorio en un 30\% lo que permitiría cubrir una mayor demanda que se podría traducir en beneficio económico.

\section{Referencias bibliográficas}

Agencia de Regulación y Control de Electricidad, (2016). Estadística Anual y Multianual del Sector Eléctrico Ecuatoriano, Quito: ARCONEL.

De La Bastida, R., Araguillin, R. \& Sotomayor, N., (2017). Reingeniería como metodología para el desarrollo de instrumentación virtual y aplicaciones integrales. En: XXVII Jornadas en ingeniería eléctrica y electónica. Quito: EPN, pp. 62-70.

Espín, F. y otros, 2016. Luminous Flux Variation on High-Pressure Sodium Lamps. Cuenca, UPS.

Illuminating Engineering Society of North America, (2013). IES Approved Method for the Electrical and Photometric measurement of High Intensity Discharge Lamps. New York: IESNA.

Illuminating Engineering Society, (2017). Approved Method for Total Luminous Flux Measurement of Lamps Using an Integrating Sphere Photometer. s.l.:Illuminating Engineering Society.

Instituto Ecuatoriano de Normalización , (2015). Requisitos generales para la competencia de los laboratorios de ensayos y de calibración, Quito: INEN.

Ministerio de Industrias y Productividad, (2016). Designación de Organismos de Evaluación de la Conformidad, Quito: MIPRO.

Velásquez, C. \& Espín , F., (2015). Cálculo de la incertidumbre combinada en un goniofotómetro de espejo rotante tipo $\mathrm{C}$ y una esfera de Ulbricht. I+-T+C Investigación, Tecnología y Ciencia, 1(9), pp. 29-35.

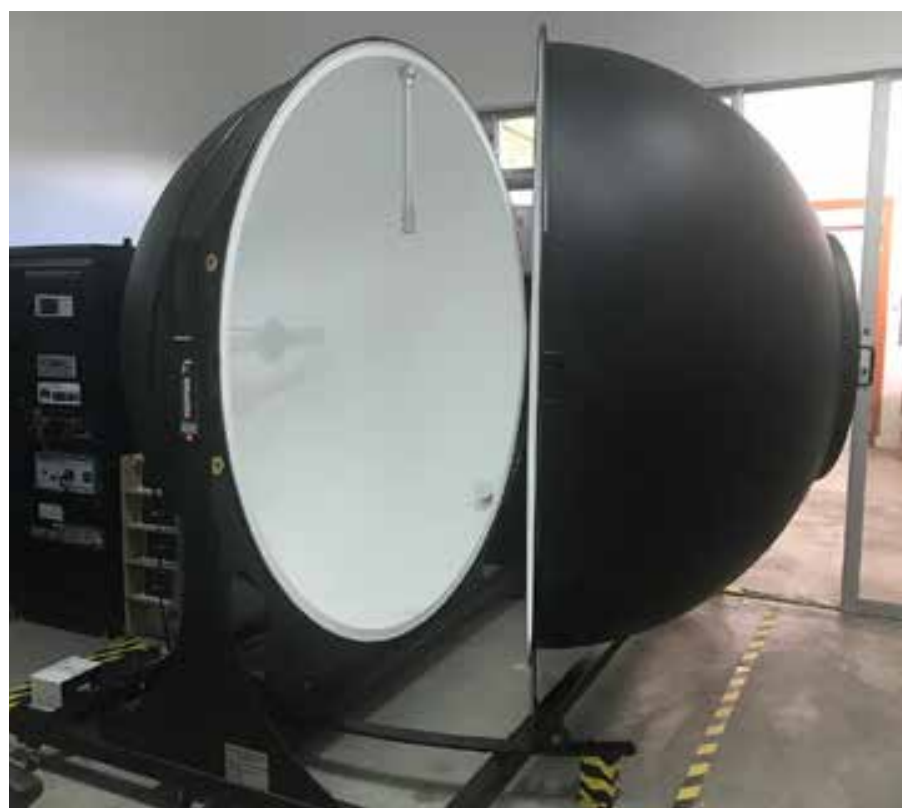

